CASO CLÍNICO

\title{
Neumoperitoneo no quirúrgico
}

\section{Non surgical pneumoperitoneum}

Paulino Martínez-Hernández-Magro ${ }^{1 *}$, José A. Lazarini-Díaz-Barriga², Javier Mendoza-Suárez ${ }^{2}$

y Gustavo Lemus-Sánchez ${ }^{3}$

${ }^{1}$ Servicio de Cirugía General y Colorrectal; ${ }^{2}$ Servicio de Geriatría; ${ }^{3}$ Servicio de Imagenología. Hospital Guadalupano de Celaya, Celaya, Guanajuato, México

\section{RESUMEN}

Objetivo: Presentar un caso de neumoperitoneo no quirúrgico secundario a neumatosis intestinal. Presentación del caso: Hombre de 85 años de edad con síntomas gastrointestinales difusos. Durante su evaluación se encuentra aire libre perihepático en la tomografía axial computarizada (TAC) abdominal, por lo que se solicita valoración quirúrgica. Paciente asintomático y sin datos de sepsis. La TAC demuestra quistes llenos de aire intramurales y serosos en el colon, lo que sugiere el origen del neumoperitoneo. El paciente fue solo vigilado con tratamiento conservador. Discusión: La causa intraabdominal más común de neumoperitoneo espontáneo es la neumatosis quística intestinal. Conclusiones: Existen casos de neumoperitoneo no quirúrgico.

Palabras clave: Neumoperitoneo. Neumatosis intestinal. Tratamiento conservador.

\section{ABSTRACT}

Objective: To present a case of non-surgical pneumoperitoneum secondary to pneumatosis intestinalis. Case presentation: Male patient 85 years old in study for diffuse gastrointestinal symptoms, during his evaluation was found free perihepatic air in abdominal computed tomography (CT) for which surgical evaluation is requested. The patient was asymptomatic and without sepsis data. The CT scan shows intramural and serous air filled cysts in colon that suggests the origin of the pneumoperitoneum. The patient was only monitored and conservative treatment was carried out. Discussion: The most common intraabdominal cause of spontaneous pneumoperitoneum is Pneumatosis cystoides intestinalis. Conclusions: There are cases of non-surgical pneumoperitoneum.

Key words: Pneumoperitoneum. Pneumatosis intestinalis. Conservative treatment. 


\section{INTRODUCCIÓN}

El término neumoperitoneo se refiere al signo radiológico determinado por la presencia de aire libre, localizado anormalmente en la cavidad peritoneal ${ }^{1}$. Generalmente, este hallazgo sugiere una complicación intraabdominal grave y requiere tratamiento quirúrgico de urgencia. Se reportan entre un 5 a $14 \%$ de pacientes con neumoperitoneo espontáneo que puede ser manejado no quirúrgicamente ${ }^{1}$.

La fuente más común de neumoperitoneo espontáneo no quirúrgico es el originado por vía intratorácica (ventilación mecánica, barotrauma, enfermedad pulmonar obstructiva crónica (EPOC), tuberculosis, contusión pulmonar $)^{2}$, pero también hay causas intraabdominales, la causa más común de neumoperitoneo espontáneo es la neumatosis quística intestinal.

El objetivo de presentar este caso es mostrar un paciente con neumoperitoneo no quirúrgico, que el cirujano debe manejar conservadoramente.

\section{PRESENTACIÓN DEL CASO}

Se trata de un paciente de sexo masculino, de 85 años de edad, que es evaluado por el Departamento de Geriatría por malestar abdominal inespecífico con distensión y estreñimiento. Durante la evaluación, el paciente es sometido a una tomografía axial computarizada (TAC) abdominal, en la que se encuentra aire libre intraabdominal de predominio perihepático, motivo por el cual se solicita valoración quirúrgica por sospecha de colecistitis enfisematosa. Al evaluar al paciente se encuentra consciente, orientado, con adecuada coloración de tegumentos, TA 110/60 mmHg, FC $78 x^{\prime}$, temperatura $36.5^{\circ} \mathrm{C}$, abdomen blando, depresible, no doloroso a la palpación profunda, no se palpan plastrones ni masas intraabdominales, presenta peristálsis normal, y no hay datos objetivos de irritación peritoneal.

Pruebas de laboratorio con $\mathrm{Hb}$ de $12.7 \mathrm{~g} / \mathrm{dl}$,

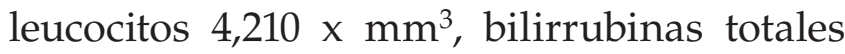
$0.70 \mathrm{mg} / \mathrm{dl}$, BD $0.33 \mathrm{mg} / \mathrm{dl}, \mathrm{BI} 0.37 \mathrm{mg} / \mathrm{dl}$, TGO 21 UI/1, TGP 17 UI/1, antígeno carcinoembrionario $4.24 \mathrm{ng} / \mathrm{ml}$. Al revisar la TAC abdominal se aprecia la presencia de aire libre perihepático en corte coronal (Fig. 1) y en corte axial (Fig. 2), y en el colon se observan lesiones quísticas intramurales llenas de aire, de predominio en el colon izquierdo (Figs. 3 y 4), con lo que se concluye que la causa de neumoperitoneo espontáneo fue la ruptura de alguno de estos quistes. Al encontrarse el paciente estable hemodinámicamente $y$ sin datos de sepsis, se decide manejarlo ambulatoriamente y bajo vigilancia, y solamente agregar fibra (psillium plantago) a la dieta para disminuir la presión intracolónica. El paciente, al seguimiento, continua asintomático y ha mejorado su patrón evacuatorio, por lo que fue dado de alta de la Consulta de Cirugía y continua su seguimiento en el Departamento de Geriatría.

\section{DISCUSIÓN}

Generalmente, el término neumoperitoneo implica una condición intraabdominal grave que requiere de cirugía de urgencia ${ }^{3}$. Se ha reportado también la presencia de neumoperitoneo sin evidencia de perforación visceral 


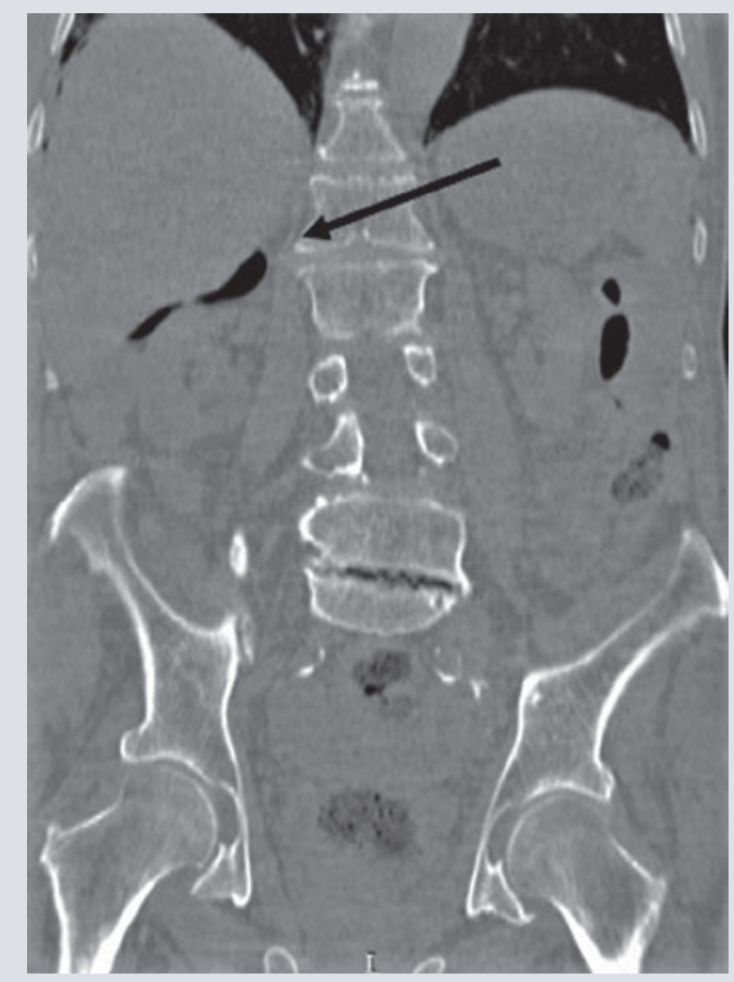

Figura 1. Presencia de aire libre perihepático en corte coronal (flecha).

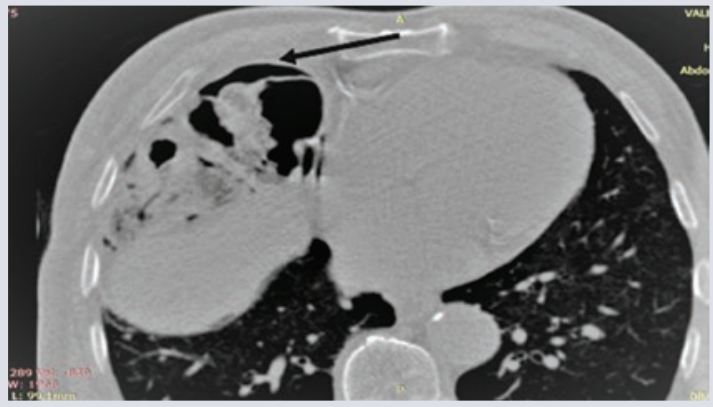

Figura 2. Presencia de aire libre en corte axial (flecha).

en un 5 a 14\% de los casos. En algunas series se reportan hasta un $44 \%$ de cirugías en pacientes con neumoperitoneo no quirúrgico en las que no se evidenció perforación intestinal ${ }^{1}$.

Tradicionalmente, la ruta de un neumoperitoneo espontáneo es la torácica (en pacientes

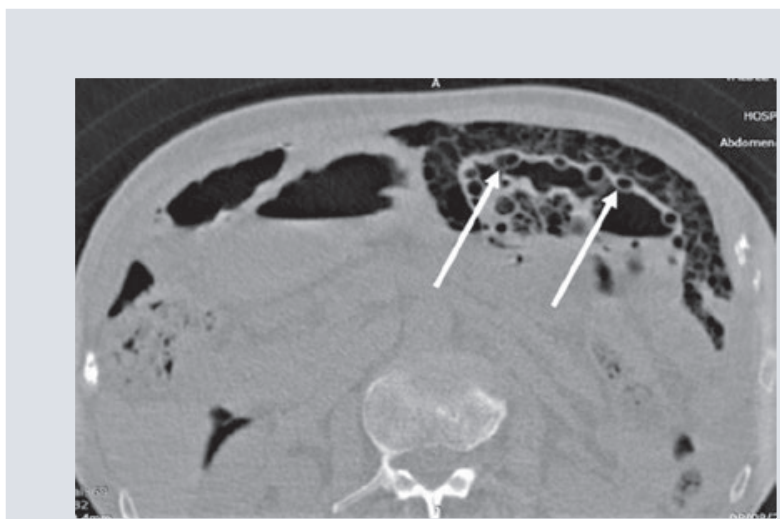

Figura 3. Neumatosis intestinal en corte axial y aire intramural (flechas).

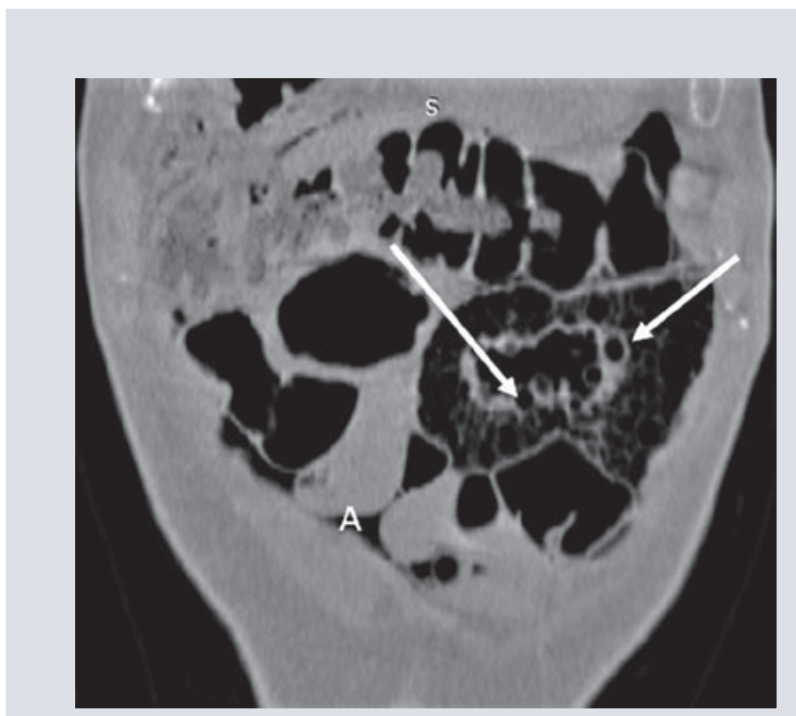

Figura 4. Neumatosis intestinal (flechas). A: Aire libre intraperitoneal.

sometidos a ventilación positiva, pacientes con EPOC, tuberculosis pulmonar, asma, ruptura espontánea de bulas, trauma torácico cerrado) ${ }^{1,4}$ e, incluso, en pacientes después de reanimación cardiopulmonar, debido al aumento de la presión en la vía aérea, así como la necesidad de aplicar compresiones al tórax ${ }^{1,5}$.

La causa intraabdominal más común de neumoperitoneo espontáneo es la neumatosis 
quística intestinal ${ }^{1}$. La neumatosis intestinal se refiere a la presencia de aire intramural en el intestino, y cuando está limitada al colon se nombra neumatosis coli ${ }^{6-8}$. El aire puede estar localizado en quistes en la submucosa, subserosos o, inclusive, en la muscularis propia ${ }^{7,9}$. La neumonitis quística intestinal generalmente puede estar en conjunto con algunos otros padecimientos, como la EPOC o la enfermedad intestinal inflamatoria. Todo padecimiento torácico que introduzca aire vía mediastinal puede originar también neumatosis intestinal ${ }^{1}$.

Los síntomas son inespecíficos, incluyen dolor abdominal, distensión, estreñimiento, diarrea hemorrágica, tenesmo ${ }^{1,7}$. El hallazgo puede ser incidental al realizar estudios de imagen $^{10}$.

La mejor herramienta diagnóstica es la TAC ${ }^{6}$. La colonoscopia puede ser diagnóstica, sin embargo, a veces pueden confundirse las lesiones quísticas con pólipos ${ }^{11}$.

No hay una causa específica en la etiología de la neumatosis, dentro de las causas de este padecimiento se han identificado algunos medicamentos, como corticoesteroides e inmunosupresores, sin embargo, puede estar relacionada con enfermedades pulmonares, gastrointestinales, infecciosas y autoinmunes ${ }^{6}$. La teoría mecánica propone que el aumento en la presión intraluminal fuerza el paso del gas intraluminal a la pared intestinal $^{10}$. Existen casos documentados de neumatosis coli posterior a preparación colónica para colonoscopia ${ }^{12}$.

La presencia concomitante de gas portal y sepsis son factores asociados con alta mortalidad $^{6}$.
Cuando se identifica una causa específica, el tratamiento va encaminado a la causa de origen (p. ej., enfermedad de Crohn) o suspender el medicamento que induce su formación ${ }^{6}$. En el paciente estable hemodinámicamente y con exploración abdominal normal, el tratamiento no quirúrgico es el apropiado ${ }^{3,6}$, la neumatosis coli idiopática generalmente sigue un curso asintomático, y la reabsorción del gas ocurre con el tiempo ${ }^{11}$, el tratamiento quirúrgico se reserva para los pacientes con signos de perforación, abscesos o isquemia intestinal ${ }^{11}$.

\section{CONCLUSIONES}

Existen cuadros de neumoperitoneo no quirúrgico en los cuales el tratamiento debe ser conservador, una causa intraabdominal puede ser la neumatosis intestinal. Una buena correlación entra estudios diagnósticos y la clínica evitará laparotomías innecesarias.

\section{CONFLICTO DE INTERESES}

Los autores certificamos que no existe conflicto de intereses.

\section{FINANCIAMIENTO}

No existió financiamiento para este caso.

\section{RESPONSABILIDADES ÉTICAS}

Protección de personas y animales. Los autores declaran que para esta investigación no se han realizado experimentos en seres humanos ni en animales. 
Confidencialidad de los datos. Los autores declaran que han seguido los protocolos de su centro de trabajo sobre la publicación de datos de pacientes.

\section{Derecho a la privacidad y consentimiento} informado. Los autores han obtenido el consentimiento informado de los pacientes y/o sujetos referidos en el artículo. Este documento obra en poder del autor de correspondencia.

\section{BIBLIOGRAFÍA}

1. Mularski RA, Ciccolo ML, Rapapport WD. Nonsurgical causes of Pneumoperitoneum. WJM. 1999;170(1):41-6.
2. Lida A, Naito H, Tsukahara K, Yumoto T, Nosaka N, Kawana S, et al. Pneumatosis cystoides intestinalis presenting as pneumoperitoneum in a patient with chronic obstructive pulmonary disease: a case report. J Med Case Reports. 2017;11:55.

3. Rachapalli W, Chaluvashetty SB. Pneumatosis Cystoides Intestinalis. J Clin Diag Res. 2017;11(6):TJ01-2.

4. Cruz-Ramos J, Pérez-Fernández LF, Herrera-García W, Granados-Navas FJ. Neumoperitoneo causado por ventilación mecánica asistida. Acta Pediatr Mex. 2010;31(4):168-73

5. Larrañaga I, Meneu JC, Díaz G, Mendía E, Rey A, Fresneda V. Neumoperitoneo no quirúrgico. Cir Esp. 2000;67:5:411-523.

6. Kelly GS, Grandy B, Rice J. Diffuse pneumatosis coli. J Emerg Med. 2018;54(6):e137-e139.

7. Nishimura JM, Farzaneh T, Pigazzi A. Pneumatosis coli causing pneumoperitoneum. J Surg Case Reports. 2017;1:1-3.

8. Iriarte Gallego G, Baez García J, Martinez Hernandez Magro P, Gomez Jardon JA, Valdéz Martinez E, Sanchez Vazquez G. Neumatosis Coli. Rev Gastroenterol Mex. 2007;72(2):133-5.

9. Vassiliou MC, Adrales GL. Pneumatosis Cystoides Coli. N Engl J Med. 2009;361:8.

10. Ooi SMS. Pneumoperitoneum in a non-acute abdomen-pneumatosis cystoides intestinalis. Surg Case Reports. 2015;1:44.

11. Mourra N, Fontugne J. Extensive pneumatosis coli misdiagnosed and mismanaged as polyposis. Scand J Gastroenterol. 2013;48:119-20.

12. Rath T, Roeb E, Doppl WE. Pneumatosis coli as a rare complication of bowel preparation. Endoscopy. 2010;42:E344-5. 